\title{
Framework for dynamic life critical situations using agents
}

\author{
Jenny Lundberg ${ }^{1}$, Anne Håkansson ${ }^{2}$ \\ ${ }^{1}$ Blekinge Institute of Technology \\ Box 520, 37225 Ronneby, Sweden \\ ${ }^{2}$ Computer and Systems Sciences \\ Forum 100, SE-164 40 Kista,Sweden \\ jlu@bth.se, anneh@dsv.su.se
}

\begin{abstract}
In this paper, we present a framework incorporating a multi-agent system (MAS) that enables aid for international effect-based operation in emergency situations. The outcome is to empower emergency personnel, which can support collaboration between different international services by informing them about the emergency, matching competences and resources of the teams and volunteers. The challenge in emergency contexts is the abbreviations forming an information-carrying structure, which is especially important when abbreviations are exchanged between different services like rescue, military and emergency. We propose a framework, which provides the right information, rescue team, and services at the right place. The MAS can support information dissemination in dynamic situations in context, based on the information extraction and matching of the contents of the underlying ontologies. In the framework the system poses a sensible solution to the international rescue teams' need of a high quality handling of life critical situations.
\end{abstract}

Keywords: Multi-agent systems, intelligent agents, emergency

\section{Introduction}

International support in emergency services is necessary to handle natural catastrophes like the earthquake creating a Tsunami in the Indian Ocean 2004. Many countries were suffering from this catastrophe, with approximately 223.000 fatalities, mainly due to citizens were taking vacation on the coasts of South-East Asia. The rescue actions from the Swedish government failed and from an investigation it was found that it suffered criticism due to the lack of an organisation managing such serious crisis. But the criticism also concerned lack of understanding the extent of the disaster, the collection of relevant information and coordination of measures [1]. Presumably, a closer connection between international rescue organisations in the 
involved countries can build a ground for a solid unified action of accurate force to meet disasters that pose immediate negative effects on several countries. Therefore, we need proper ICT systems to support disaster relief [10]. Multi-agent systems in emergency contexts have been used [2] [11] [12], but it is desirable to develop a framework with agents and ontologies to handle dynamic emergency contexts.

To receive comprehensive information and disseminate it fast, the emergency operators write abbreviations in the system, which is translated into for nationally rescue teams, commonly utilised and understandable language. This language needs to be interpreted by international rescue teams. We suggest using Multi-agent system (MAS) to support the dissemination of the information to international teams. From the input of comprehensive information to MAS, the agents handle the conversion of abbreviations into understandable language and translations into other languages.

In dynamic emergency areas, the use of acronym and abbreviations are widely spread, such as, Air Traffic Control, operator control of critical infrastructures and several other medical applications [9]. Abbreviations are context sensitive [13], and as early as 1975, Woods [3] stated the importance of semantics and not only syntax. In this paper, we work with an awareness of syntax and semantics as we apply multiagent systems in the real world context of international emergency handling.

\section{Scenario}

To develop MAS, we look at a future scenario based on the real world Tsunami 2004: Sweden and other related countries become alerted about the Tsunami through the early warning systems, and establish contacts with Thailand's SOS centres, simultaneously as the earthquake starts, which is approximately 2 hours before the wave hit the shore. Sweden sends Jumbo jets with rescue service and nursing staff, and communicates with Thailand via international language on board the Jumbo jet. They plan the rescue action and make strategies for the rescue, match competences and communicate with local rescue services. When the Swedish rescue force arrives, vehicles are waiting for them for quick transport to rescue areas, hospitals and collapsed buildings. Swedish SOS (at the scene and in Sweden) must control which other international resources are available and match their recourses and competences. They must also identify a large number of volunteers without rescue education. These will be given quick training on place. Since it is a tsunami this training is lead by rescue competent personnel. The matching of rescue competence against the requirements in the action plan is made in a unified process including search for proper rescue leading competences. Furthermore, when all the rescue teams arrive on the scene of disaster, the cooperation is established and will be maintained with responsible person in charge.

\section{The Multi-Agent System}

The MAS need to handle several different data representation forms and solve problems quickly. Each agent has a task to accomplish but a result is reached when 
several agents have found their piece of the information, which is assembled to constitute a result. The task for the agents in our research is to inform, search, match and group information in different parts in the framework, see Figure 1, to be able to combine the extracted information to get more comprehensible and understandable information. The ontologies form a framework for the agents to act upon, selecting the most suitable ontology matching the intention of the agent. The abbreviations are a natural part in the ontologies, however to be treated with specific concern. When extended information is needed, the MAS provide a possibility to search and find it externally, i.e., the web.

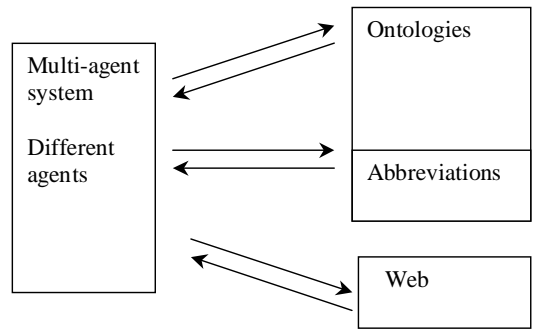

Figure 1: Simplified architecture of the multi-agent system.

The task is performed in episode in which the agents perform a single task. The call to the system is divided into parts, and each part is processed by an agent. For each time the system is invoked with new or more information, the agents must find solutions according to that information. Finding several solutions can granulate the output, which in comparison can support the system to give more accurate output that is more correct according to the situation. In these situations, where searching can be simplified and speeded up using several agents, multi-agent systems are useful [4] [5]. The agents in the multi-agent system must coordinate information from several different sources from which they can produce and deliver more complex information in right time to the right place [6], to the appropriate users. The output is the description of the critical situation in international language, as well as, priority of the cases. The information is directives to the rescue services, from which the services can evaluate the situation and determine what kind of service is needed at the scene.

Agents can allow searching for information in several different ontologies and support reasoning with the findings [7]. We need this distributed problem solving to search for information in ontologies and priorities in the cases. The agents search for information from several different ontologies and correlate it to match the context of the scene. The international abbreviations are the same, but the interpretation of them, leading to different actions. As for example a drowning situation requires rescue service action in the form of finding, lifting up the human and securing the area preventing other injuries. The ambulance service focuses on Heart-Lung-Rescue, and on giving proper drugs to save the persons life. 


\section{The Agents}

We suggest intelligent agents that learn by being sensible to new or changed environments in combination with using meta-agents. The meta-agents are built on the agents linked to the ontologies, constituting high-level connections between different possible definitions, and matching them to the information collected for the case. The system is launched by the input, which is performed by the agents needed for the task. All the types of agents are invoked to perform their assignment see Figure 2. The "inform" agents work with information and matching the abbreviations to their corresponding words and notify the rescue teams. Some words are explaining seriousness in the case and therefore, some words are used for prioritising.

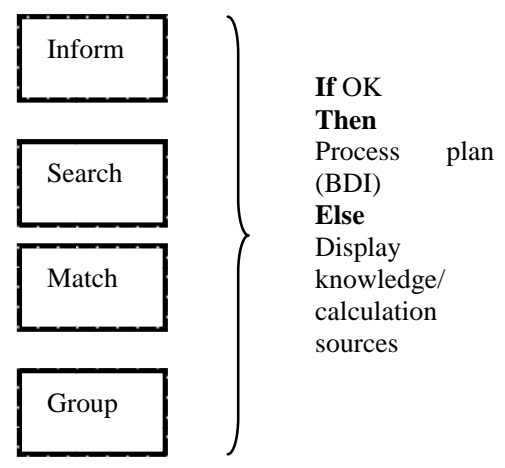

Figure 2: Inform, search, group and match agents, implemented by BDI model.

The "Search agents" search for competence and other teams to work with and "Match" agents perform matching to find right competences for right situations. The agents collect and build up information, which is describing the life critical situations corresponding to the information from the emergency call.

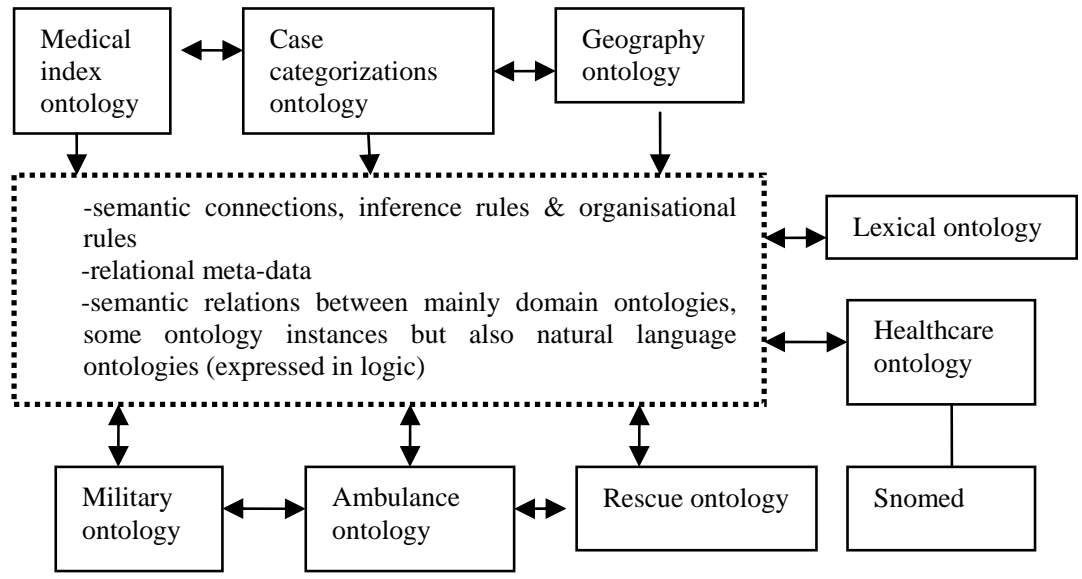

Figure 3: High-level architecture of ontologies. 
In emergencies several ontologies are to be used, see Figure 3. The agents' combination of the content of the ontologies determines the situations in which the emergency has arisen. They consist of types, properties and relationships are the knowledge foundation on which the agents perform their work. If a person have a heart stop and do not breathe, Heart-Lung-Rescue actions are to be initiated. From the information, the required services with specific equipment are called. If suitable, the meta-data learn the combination and can be reused for future combinations. These meta-agents are built on the successful agents' performances. Successful agents are the agents that give correct information according to emergency services.

In the organizational ontology that is depicted as the military, ambulance, rescue, and healthcare ontology in figure 3, the different parts of the organization are defined and interpreted with the semantic connections including organizational rules. The produced outcome by the agents as they act upon the ontology is: the rescue team that can handle an ambulance case, and the number of operators that should cooperate in handling the accidents. The ontologies are based on measurement of behaviors, such as operator coordination patterns. Another part is structure, which is based on operators own tagging of related information, as used in web 2.0. And example cases of normative behavior are included.

The medical index ontology contains of index with instructions about the support that should be given to minimize possible injuries as consequence to the event. For example, the instructions can be how the operator can give Heart-Lung-Rescue support via phone, or which special precautions to take if the person has got diabetes. In the categorization ontology, there are structures for how the cases are to be categorized (not to be confused with the ontologies relationships). In the geographical ontology there is special support for geographical spots that can have several different names, such as a town park. A town park can have several names, a name on the map, a popular name, and yet another name of the park in relation to its location or surroundings. Alignments tools such as minimal mappings can preferably be used however validations assuring correct connection to the domain need to be performed.

In the categorization ontology the abbreviations are defined. The example of an abbreviation is: H1.11.23. For the trained operator H1.11.23 is: Human, highest priority, animal bite, and unconscious, which mean that a specific ambulance, with correct equipment and geographical location has to be dispatched. In the healthcare ontology, organisational healthcare rules are defined. Snomed ${ }^{3}$ [8], a national specialist language for healthcare is connected. The multi-agent system works with ontologies, internally in the system, to find words and produce messages corresponding to the abbreviations, and, externally at the Web, to find geographic location with waved ground. Relations and rules that are not already defined and connected are stored in a database and then further used and updated in suitable ontology. Furthermore, the evaluation of the result of the agents is made in relation to the debriefing session, were also the actions of the agents are collected and evaluated.

\footnotetext{
${ }^{3}$ Thus, Snomed is not an ontology but a human readable conception system, which (most probably) can form a solid basis for a machine readable ontology.
} 


\section{Conclusions}

In this paper, we provided a multi-agent technology to support international handling of life critical cases. The agents act upon input data from the scene of accident, and match information from the ontologies, which are processed to meet the demand. The agents search, match, group and inform the rescue team about the situation, and are implemented by using BDI model. This article is a contribution to the empowering of emergency personnel in life critical contexts performing effect-based operations on an international arena.

\section{References}

1. http://www.riksdagen.se/webbnav/index.aspx?nid=3281\&dok_id=GTB3104d1

2. Lundberg, J., and Håkansson, A., (2008). An Approach towards using Agent in Multi-Agent Systems to streamline emergency services. In Proceedings of 5th International Conference on Information Technology and Applications, Cairns, Queensland, AUSTRALIA.

3. Woods, W. A., "What's in a link: Foundations for semantic networks," in D. G. Bobrow and A. M. Collins (Eds). Representation and Understanding. New York: Academic Press, 1975.

4. Shoham, Y and Leyton-Brown, K. (2008) Multiagent Systems: Algorithmic, GameTheoretic, and Logical Foundations, Cambridge University Press, ISBN 9780521899437.

5. Bratman, M. E. [1987] (1999). Intention, Plans, and Practical Reason. CSLI Publications. ISBN 1-57586-192-5

6. Apelkrans M. and Håkansson, A., 2008. Information coordination using Meta-Agents in Information Logistics Processes. In Proceedings of Knowledge-Based and Intelligent Information \& Engineering Systems, LNCS/LNAI, Springer-Verlag, pp. 788-798.

7. Hartung, R., and Håkansson, A., 2008. Using Meta-agents to reason with Multiple Ontologies. In Proceedings of Agent and Multi-Agent Systems: Technologies and Applications Agent and Multi-Agent Systems: Technologies and Applications, LNCS/LNAI, Springer-Verlag, pp. 261-270.

8.http://www.socialstyrelsen.se/Amnesord/inter_fragor/SnomedCT/specnavigation/Aktuellt/akt uellt.htm

9. Pakhomov, Serguei, Semi-supervised maximum entropy based approach to acronym and abbreviation normalization in medical texts, ACL 02, Proceedings of the $40^{\text {th }}$ annual meeting on association for computational linguistics, 2001

10. Denning, P. J. Hastily formed networks, Com. of the ACM, vol. 49, No 4, April, 2006

11. Molina, M., and Blasco, G.,"A Multi-agent System for Emergency Decision Support," In: Intelligent Data Engineering and Automated Learning Book. Lecture Notes in Computer Science. Springer Berlin/ Heidelberg. Vol .2690/2003, 2003, pp. 43-51.

12. Schoenharl,T., Madey, G. Szabó, G., and Barabási, A-L.,"WIPER: A multi-agent system for emergency response." In Proceedings of the 3rd International community on information systems for crises response and management Conference, Newark, NJ, USA, 2006.

13. Lundberg, Jenny, Gustavsson Rune, Robust approach towards context dependant information sharing in distributed environments, ICEIS09, Milan, Italy, 2009 